\title{
Article
}

\section{The X-Ray Variable Sky as Seen by MAXI: The Future of Dust-echo Tomography with Bright Galactic X-Ray Bursts}

Corrales, Lia, Mills, Brianna S., Heinz, Sebastian and Williger, Gerard

Available at https://clok.uclan.ac.uk/28404/

Corrales, Lia, Mills, Brianna S., Heinz, Sebastian and Williger, Gerard (2019) The X-Ray Variable Sky as Seen by MAXI: The Future of Dust-echo Tomography with Bright Galactic X-Ray Bursts. The Astrophysical Journal . ISSN 0004-637X

It is advisable to refer to the publisher's version if you intend to cite from the work. http://dx.doi.org/10.3847/1538-4357/aboc9b

For more information about UCLan's research in this area go to http://www.uclan.ac.uk/researchgroups/ and search for <name of research Group>.

For information about Research generally at UCLan please go to http://www.uclan.ac.uk/research/

All outputs in CLoK are protected by Intellectual Property Rights law, including Copyright law. Copyright, IPR and Moral Rights for the works on this site are retained by the individual authors and/or other copyright owners. Terms and conditions for use of this material are defined in the policies page. 


\title{
The X-Ray Variable Sky as Seen by MAXI: The Future of Dust-echo Tomography with Bright Galactic X-Ray Bursts
}

\author{
Lia Corrales (D) ${ }^{1,2,8,9}$, Brianna S. Mills ${ }^{2,3,4}$ (D) Sebastian Heinz ${ }^{2}$ (D), and Gerard M. Williger ${ }^{3,5,6,7}$ \\ ${ }^{1}$ University of Michigan, Ann Arbor, MI 48109, USA; liac@umich.edu \\ ${ }^{2}$ University of Wisconsin-Madison, Madison, WI 53706, USA \\ ${ }^{3}$ University of Louisville, Louisville, KY 40292, USA \\ ${ }^{4}$ University of Virginia, Charlottesville, VA 22904, USA \\ ${ }^{5}$ Jeremiah Horrocks Institute, University of Central Lancashire, Preston, PR1 2HE, UK \\ ${ }^{6}$ Institute for Astrophysics and Computational Sciences, Catholic University of America, Washington, DC 20064, USA \\ Konkoly Observatory, 15-17 Konkoly-Thege Miklós út, 1121 Budapest, Hungary \\ Received 2018 October 31; revised 2019 March 1; accepted 2019 March 3; published 2019 April 3
}

\begin{abstract}
Bright, short duration X-ray flares from accreting compact objects produce thin, dust scattering rings that enable dust-echo tomography: high-precision distance measurements and mapping of the line-of-sight distribution of dust. This work looks to the past activity of X-ray transient outbursts in order to predict the number of sight lines available for dust-echo tomography. We search for and measure the properties of $3 \sigma$ significant flares in the 2-4 keV light curves of all objects available in the public MAXI archive. We derive a fluence sensitivity limit of $10^{-3} \mathrm{erg} \mathrm{cm}^{-2}$ for the techniques used to analyze the light curves. This limits the study mainly to flares from Galactic X-ray sources. We obtain the number density of flares and estimate the total fluence of the corresponding dust echoes. However, the sharpness of a dust-echo ring depends on the duration of a flare relative to quiescence. We select flares that are shorter than their corresponding quiescent period to calculate a number density distribution for dust-echo rings as a function of fluence. The results are fit with a power law of slope $-2.3 \pm 0.1$. Extrapolating this to dimmer flares, we estimate that the next generation of X-ray telescopes will be 30 times more sensitive than current observatories, resulting in 10-30 dust ring echoes per year. The new telescopes will also be 10-100 times more sensitive than Chandra to dust ring echoes from the intergalactic medium.
\end{abstract}

Key words: dust, extinction - scattering - X-rays: binaries - X-rays: bursts - X-rays: ISM

\section{Introduction}

Interstellar dust scatters X-ray light over arcminute-scale angles, producing a diffuse scattering halo with an integrated flux $F_{\text {halo }}(E)=F_{a}\left(1-e^{-\tau}\right)$, where the optical depth to X-ray scattering, $\tau \approx 0.5 E_{\mathrm{keV}}^{-2}\left(N_{\mathrm{H}} / 10^{22} \mathrm{~cm}^{-2}\right)$, and $F_{a}$ is the absorbed flux of the central X-ray source (Predehl \& Schmitt 1995; Corrales et al. 2016). Because the scattered light takes a longer path to reach the observer, the observed scattering halo surface brightness profile is a convolution of the dust's line-of-sight position, grain size distribution, and $F_{a}(t)$ light curve (Heinz et al. 2015, and references therein). When $F_{a}(t)$ takes the form of a single burst with high amplitude and short duration, a scattering halo will appear as a set of discrete rings, where each ring corresponds to a different foreground dust cloud. These rings expand with a characteristic $t^{1 / 2}$ time dependency that allows X-ray astronomers to map the line-ofsight distribution of dust ("dust-echo tomography") to much higher resolution than is currently available with any other method (Trümper \& Schönfelder 1973; Heinz et al. 2015, 2016).

Mapping the interstellar medium (ISM) through dust-echo tomography is also important for interpreting the time and spectral evolution of accreting compact objects. Dust echoes are known to affect the spectral evolution of X-ray variable objects, producing a prolonged soft-tail (e.g., Pintore et al. 2017a; Jin et al. 2018). This confusion is particularly acute for

\footnotetext{
8 University of Michigan LSA Collegiate Fellow.

9 Einstein Postdoctoral Fellow.
}

$\mathrm{X}$-ray timing missions with low imaging resolution: RXTE, MAXI, and NICER.

To date, the brightest dust-echo rings observed have come from four Galactic X-ray sources-1E 1547.0-5408 (Tiengo et al. 2010; Olausen et al. 2011; Pintore et al. 2017a), Cir X1 (Heinz et al. 2015), V404 Cygni (Beardmore et al. 2016; Heinz et al. 2016; Vasilopoulos \& Petropoulou 2016), and 4U 1630-47 (Kalemci et al. 2018). Dust echoes can also be produced by the X-ray components of gamma-ray bursts (GRBs), which scatter off the nearby Galactic medium (Vaughan et al. 2004, 2006; Tiengo \& Mereghetti 2006; Vianello et al. 2007; Pintore et al. 2017b). Table 1 lists the approximate soft X-ray fluence and ISM column for dust-echo rings observed around GRBs and XRBs. In most of the GRB cases, fluences were measured from the properties of the dust scattering echo, and the results depend on the adopted grain size distribution.

One can note from Table 1 that the fluences of GRBs producing dust echoes are particularly low. In these cases, $\sim 10 \%$ of the X-ray light from the flare is deposited into the dust scattering ring. This level rivals the amount of light in the telescopes' point-spread function wings. However, due to the quick dimming typical of X-ray afterglows, the time delay between the prompt X-ray emission and the dimming afterglow allows the the dust scattering rings to stand out in contrast, even when a central X-ray point source is visible. In theory, X-ray variability from any high-redshift object can produce echoes that propagate off dust from foreground galaxies or the intergalactic medium (IGM), but this requires more sensitive 
Table 1

Flare Properties of Objects with Observed Dust Echoes

\begin{tabular}{|c|c|c|c|c|}
\hline Object & Telescope(s) & $\begin{array}{l}\text { Fluence }^{\mathrm{a}} \\
\left(\mathrm{erg} \mathrm{cm}^{-2}\right)\end{array}$ & $\underset{\left(10^{22} \mathrm{~cm}^{-2}\right)}{\text { Gal. } N_{\mathrm{H}}}$ & References \\
\hline GRB 031203 & XMM-Newton & $(0.7-3) \times 10^{-6}$ & 0.6 & $\begin{array}{l}\text { Vaughan et al. (2004), Watson et al. (2006) } \\
\text { Tiengo \& Mereghetti (2006) }\end{array}$ \\
\hline GRB 050713A & XMM-Newton & $5 \times 10^{-7}$ & 0.1 & Tiengo \& Mereghetti (2006) \\
\hline GRB 050724 & Swift & $2 \times 10^{-7}$ & 0.6 & Vaughan et al. (2006) \\
\hline GRB 061019 & Swift & $5 \times 10^{-7}$ & 0.9 & Vianello et al. (2007) \\
\hline GRB 070129 & Swift & $7 \times 10^{-7}$ & 0.1 & Godet et al. (2007), Vianello et al. (2007) \\
\hline GRB $160623 \mathrm{~A}$ & XMM-Newton & $2 \times 10^{-6}$ & 0.7 & Pintore et al. (2017b) \\
\hline 1E $1547.0-5408$ & Swift, XMM-Newton & $(2-6) \times 10^{-3}$ & 3.0 & Tiengo et al. (2010), Halpern et al. (2008) \\
\hline Cir X-1 & Chandra & 0.025 & 2.0 & Heinz et al. (2015) \\
\hline V404 Cygni & XMM-Newton, Chandra & 0.01 & 0.6 & Heinz et al. (2016) \\
\hline 4U $1630-47$ & Swift, Chandra & 0.015 & 13.0 & Kalemci et al. (2018), estimated from MAXI \\
\hline
\end{tabular}

Note.

a $2-4 \mathrm{keV}$, absorbed fluences estimated from the references cited.

telescopes than are currently available (Miralda-Escudé 1999; Corrales \& Paerels 2012; Corrales 2015).

Many Galactic XRBs are persistent sources of X-rays, producing a quiescent dust scattering halo. A source that experiences frequent outbursts, or high variation, will create a time-variable scattering halo with no clearly defined rings. This study focuses on identifying single, large amplitude outbursts capable of producing thin, high-contrast dust-echo rings.

This work evaluates the X-ray light curves from all sources monitored regularly by the Monitor of All-sky X-ray Image (MAXI; Matsuoka et al. 2009), in order to gather the rate of X-ray flares propagating through the ISM. In Section 2, we describe the algorithm used to identify flares and discuss its limitations. In Section 3, we calculate the number distribution of flares identified in MAXI. A metric for evaluating the likelihood of an outburst to produce sharp ring echoes is discussed in Section 3.1. In Section 3.2, we fit a power law to the number distribution of flares and use it to estimate how many X-ray dust ring echoes will be seen with the next generation of X-ray telescopes. We also update the results of Corrales (2015) to estimate the number of X-ray scattering echoes that might be found arising from dust in the IGM. All findings are summarized in Section 4.

\section{Data Analysis}

MAXI is the longest operating current all-sky monitor for the soft X-ray band, which is sensitive to dust scattering. The Soft X-ray Large Angle Camera on MAXI captures almost the entire sky on a cadence of 92 minutes, over the $0.7-12 \mathrm{keV}$ energy band, with a binned one-day sensitivity limit of $4.5 \mathrm{mCrab}\left(10^{-10} \mathrm{erg} \mathrm{s} \mathrm{cm}^{-2}\right)$ (Matsuoka et al. 2009; Kawai et al. 2014). Because the ISM preferentially removes soft $\mathrm{X}$-rays, the spectral energy distribution of $\mathrm{X}$-ray scattering halos tend to peak around $1-3 \mathrm{keV}$. We use the publicly available one-day binned $2-4 \mathrm{keV}$ light curves from 398 point sources currently available on the MAXI website, ${ }^{10}$ from the start of MAXI operations to MJD 58408, to estimate the probability distribution of soft X-ray flares across the sky. For the purposes of this work, a flare is defined as any duration longer than several days for which an object's flux is $>3 \sigma$ above its dimmest state.

\footnotetext{
${ }^{10}$ http://maxi.riken.jp/top/lc.html
}

It should be noted that MAXI is insensitive to most flares that are significantly shorter than a single ISS orbit. Magnetars, Type I neutron star bursts, and X-ray afterglows to GRBs fall into this category. As will be demonstrated below, such flares are missed from this study due to limitations of MAXI, not due to data analysis choices.

\subsection{Detection Algorithm}

First, we removed all data points where a monitored object was within $10^{\circ}$ of the Sun, which is a large source of contamination. All light curves were smoothed using a threeday Gaussian convolution, to improve the stability of the algorithm. Limitations imposed by smoothing are discussed in Section 2.3.

For a baseline flux, we selected the 16th-percentile value from the distribution of flux values within the entire light curve. We then subtracted the baseline value from the light curve and calculated the signal-to-noise ratio for each bin. Flares were identified by flagging light curve intervals with a signal-tonoise ratio greater than three. Flare intervals shorter than five days were discarded. If two flares were separated by an interval shorter than five days, we combined them into one time interval. This process was repeated so that there was no quiescent period shorter than five days. These choices were motivated by analysis of a simulated data set, described in Section 2.2. The five-day cut-off significantly reduced the number of falsely identified flares.

Finally, we calibrated each light curve by normalizing them with the MAXI light curve for the Crab pulsar, which has a $2-4 \mathrm{keV}$ band flux of $1.1 \times 10^{-8} \mathrm{erg} \mathrm{cm}^{-2} \mathrm{~s}^{-1}$. We used linear interpolation over data gaps to arrive at a total fluence for each flare interval.

Figure 1 shows the results for three light curves of interest. LMC X-3 exhibits erratic behavior with no clearly defined quiescent state. The algorithm flags the intervals when LMC X3 is in a bright state. The next two panels show Cir X-1 and $4 \mathrm{U} 1630-47$ during the flares leading to dust echoes study by Heinz et al. (2015) and Kalemci et al. (2018), respectively. The calculated fluences are $0.023 \mathrm{erg} \mathrm{cm}^{-2}$ over 85 days (Cir X-1) and $0.021 \mathrm{erg} \mathrm{cm}^{-2}$ over 171 days (4U 1630-47). These values are consistent with those in the published works.

We were unable to check on the dust-echo producing flares from 1E 1547.0-5408, which occurred before the launch of 

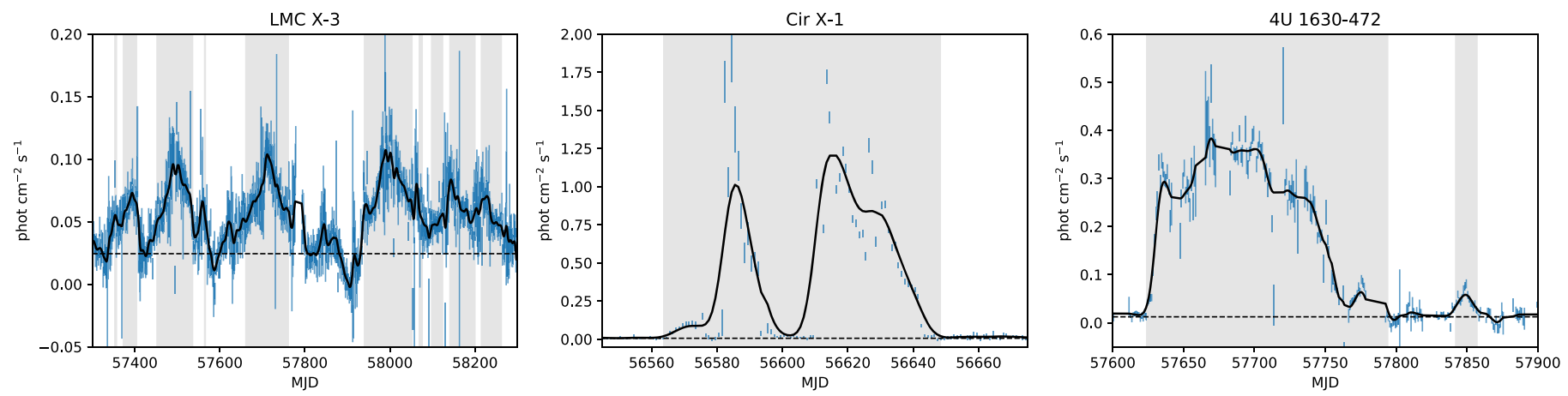

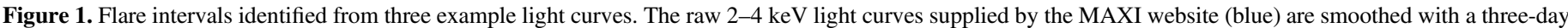

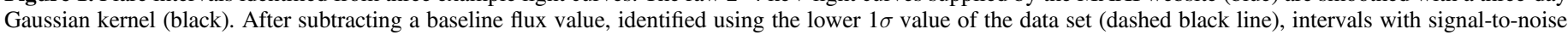

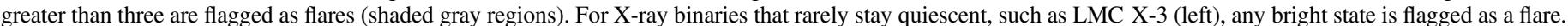

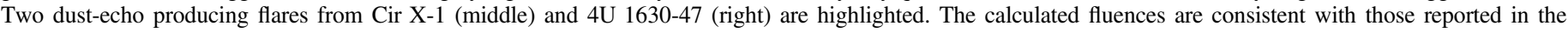
literature.

MAXI, and V404 Cygni, which was only observable by the degraded GSC3 instrument at the time of the flare (Negoro et al. 2015). The publicly available MAXI light curves do not include data from GSC3. The MAXI view of V404 Cygni is also affected by source confusion with Cyg X-1, which is usually much brighter. As a result, the light curve is poorly calibrated and no flares were measured from V404 Cygni. ${ }^{11}$

\subsection{Sensitivity Limits}

To examine the accuracy of our analysis, we simulated 1000 MAXI light curves of 900 days long, and injected one Gaussian flare into each. The reported MAXI sensitivity is 4.5 mCrab for one-day binned data (Matsuoka et al. 2009), yielding a fluence theoretical lower limit of $5 \times 10^{-5} \mathrm{erg} \mathrm{cm}^{-2}$ for a five-day long flare. As such, the flare properties were drawn from a uniform distribution of fluences, $\log \left(\mathcal{F}_{\text {cgs }}\right) \in[-5,-1] \quad$ (where $\mathcal{F}_{\text {cgs }}$ is fluence in units of erg $\mathrm{cm}^{-2}$ ), and a uniform distribution of Gaussian widths $\sigma$ (days) $\in[1,50] .{ }^{12}$ The baseline flux and error bars for each simulated light curve were drawn randomly from three MAXI light curves of objects with a quiescent flux below the sensitivity limits, i.e., those exhibiting a light curve consistent with zero flux throughout: 1ES 1101-23.2, WW Cet, and VY Ari. We take these objects as representative of the zero values and error bars arising from the MAXI calibration processes.

Figure 2 shows the distribution of detected flares compared to the input distribution. In general, the algorithm returns a large number of short $10^{-4} \mathrm{erg} \mathrm{cm}^{-2}$ flares $(<3-5$ days $)$ that appear to arise from the noise typical in the MAXI data set. We were able to cut down on the number of false positives significantly by ignoring flares shorter than five days, and by combining flares that were separated by less than five days. The dotted line in Figure 2 shows how many flares for which the fluence was correctly retrieved to within $20 \%$. For the subset of flares with fluence $>10^{-3} \mathrm{erg} \mathrm{cm}^{-2}$, our algorithm was able to identify $90 \%$ of all the simulated flares and $100 \%$ of those that were of duration $\leqslant 20$ days. We therefore take $\log \mathcal{F}_{\text {cgs }}>-3$ as the completeness limit for this study.

\footnotetext{
11 Communication with MAXI calibration staff.

12 A 900-day light curve was deemed sufficient to capture flares that are effectively 300 days long (Gaussian $\sigma=50$ days). As shown later in Section 3.1, these very long outbursts are typically beyond the scope of interest for dust-echo tomography.
}

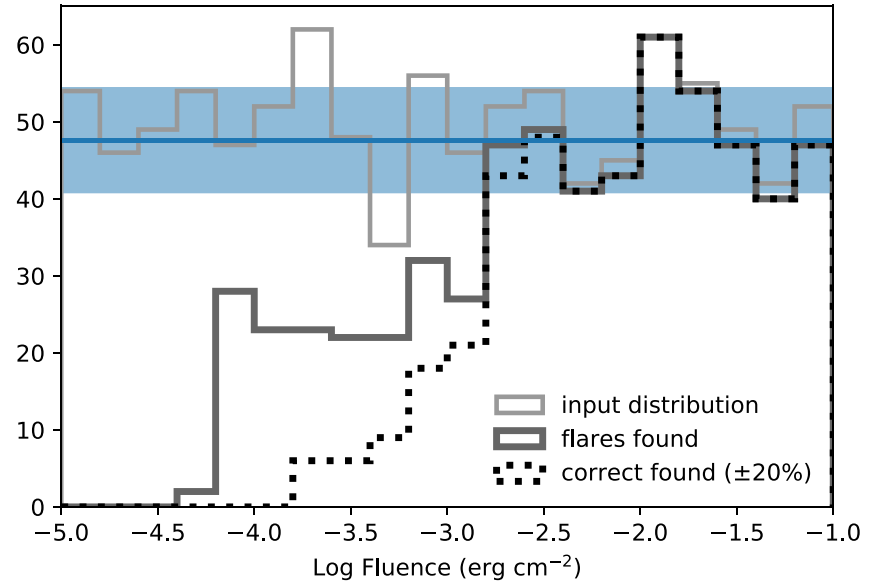

Figure 2. A histogram of simulated input (light gray) and the histogram derived with the techniques described in Section 2 (dark gray). The input flares were drawn from a uniform distribution, and the expected $1 \sigma$ variation is shaded in blue. The dotted black histogram shows how many of the output flares were correct identifications, to within $20 \%$ of the input fluence.

Identification of long, high fluence flares is limited by the flux in each bin. We estimated the flux sensitivity of our algorithm by dividing each fluence value by its corresponding duration, yielding $4 \mathrm{mCrab}$, which is consistent with the expectations for one-day binned MAXI data.

\subsection{Limitations for Short Outbursts}

Using daily binned light curves and three-day smoothing imposes selection effects against short flares. In the extreme case of a flare restricted to a single ISS/MAXI orbit (90 minutes), the signal-to-noise of the flare in one-day binned data will be reduced by a factor of $\sqrt{15} \sim 4$ compared to the singleorbit light curve. This would eliminate single-orbit flares of signal-to-noise lower than $12(<80 \mathrm{mCrab})$ from detection, corresponding to a fluence $<10^{-5} \mathrm{erg} \mathrm{cm}^{-2}$. This value is several orders of magnitude below the fluence values of interest for dust-echo tomography with Galactic X-ray sources (lower portion of Table 1), which is the main target of this study.

In conclusion, smoothing data has the advantage of reducing the number of false positives, because the variance of the data is significantly reduced. The critical metric for the detectability of dust scattering echoes of flares is their fluence, which is preserved in binning and smoothing. Section 2.2 demonstrates 

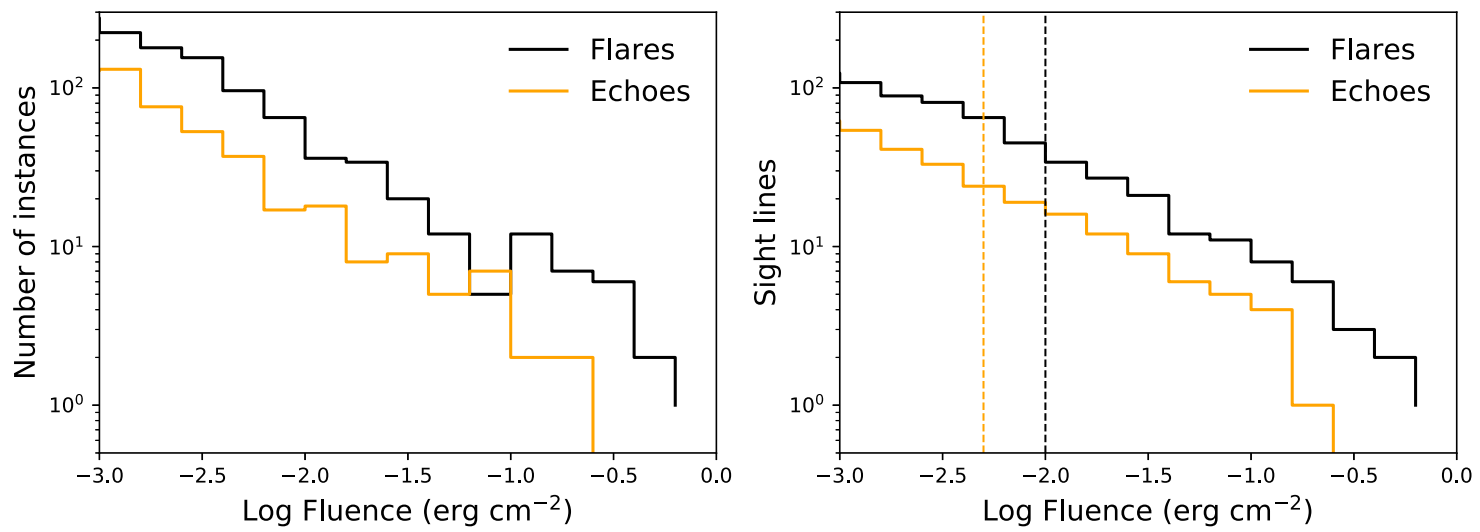

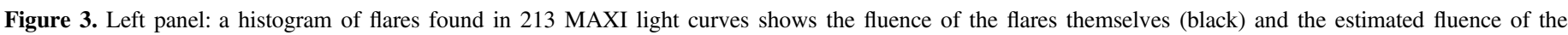

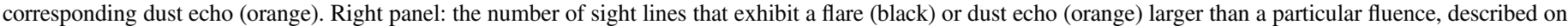

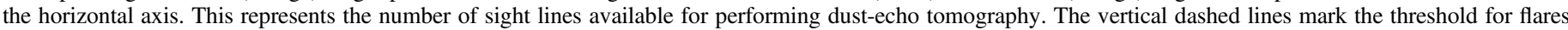

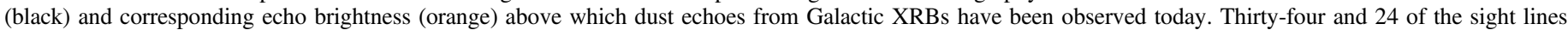
satisfy the two thresholds, respectively.

that the algorithm retrieved all of the short duration flares with $\log \mathcal{F}_{\text {cgs }}>-3$, which is an order of magnitude below our threshold of interest demonstrated by the lower portion of Table 1. Thus, the benefits of using binned and smoothed data outweigh the reduction of sensitivity to short flares.

\section{Results and Discussion}

Of the 398 sources analyzed, 213 exhibited outbursts that were picked up by the flare detection algorithm. To account for source confusion, we evaluated the light curves of three sources within $2^{\circ}$ of each other: SMC X-1, SMC X-3, and MAXI J0057-720. One flare from SMC X-3 appeared coincidentally in the light curve of MAXI J0057-720, which is 0.6 away. However, variations from SMC X-1, which is the brightest of the three and $2^{\circ}$ away from the other two objects, did not affect the light curves of either. Thus, we chose $1^{\circ}$ as the threshold for evaluating the effects of source confusion. We identified pairs of sources in the MAXI data set separated by $<1^{\circ}$. Within this subset of light curves, we searched for flares appearing within 30 days of each other. When coincident flares were found, we kept the larger fluence event and discarded the other. We also visually evaluated the light curves of sources within $2^{\circ}$ of the Galactic Center, which hosts a large number of variable compact objects that cannot be resolved with MAXI. The overall process resulted in the removal of eight flares that were double counted, leaving a total of 854 distinct outbursts with $\log \mathcal{F}_{\text {cgs }}>-3$.

Figure 3 shows a histogram of total number of flares detected as function of fluence (black). We used the Chandra X-ray Center tool colden to look up the $N_{\mathrm{H}}$ value from HI surveys, in order to estimate the optical depth of X-ray scattering at $1 \mathrm{keV}$. We then multiplied the fluence of each flare by a factor of $\left(1-e^{-\tau}\right)$ to estimate the integrated fluence of the resulting dust echo (orange).

A higher instrument background makes it difficult to observe a dust scattering halo. A small fluence (relative to the quiescent state) will produce a small perturbation in the scattering halo brightness that is unlikely to be observable. To avoid modeling the problem, we use the examples of spectacular dust ring echoes from the literature (bottom portion of Table 1) to arrive at approximate thresholds for observation with modern-day $\mathrm{X}$-ray telescopes. We chose flares with $\log \mathcal{F}_{\text {cgs }}>-2$ as candidates for producing dust-echo rings. However, some of these flares may appear bright due to having a low ISM column. At the same time, bright flares offer the chance to produce serendipitous results in high contrast. For example, V404 Cygni has $N_{\mathrm{H}} \approx 6 \times 10^{21} \mathrm{~cm}^{-2}$ or $\tau \approx 0.1$. The corresponding estimate yields the dimmest available dust-echo fluence $\left(\approx 0.006 \mathrm{erg} \mathrm{cm}^{-2}\right)$ in Table 1 , yet V404 Cygni produced some of the clearest multi-structured dust-echo rings (Heinz et al. 2016). We take $0.005 \mathrm{erg} \mathrm{cm}^{-2}$ as an approximate threshold for effective dust-echo tomography with modern-day instruments.

Because one object can produce multiple flares, we counted the number of MAXI targets that exhibited a flare fluence larger than a given threshold, yielding the number of sight lines available for dust-echo tomography (Figure 3, right). We found that 34 of the objects exhibited flares with $\log \mathcal{F}_{\text {cgs }}>-2$, and 24 of these have predicted dust echoes over the $0.005 \mathrm{erg} \mathrm{cm}^{-2}$ threshold during the last $9 \mathrm{yr}$ of MAXI operation. However, more analysis is needed to determine which of these would have produced the thin, high-contrast rings that are ideal for measuring the line-of-sight dust distribution.

\subsection{Identifying Sources of Dust-echo Rings}

High fluence flares are necessary to produce dust scattering halos, but many of them have a large fluence simply because they are long. Two other conditions are important for identifying dust-echo candidates. First, flares must be short enough to produce sharp rings. Second, the bursts must be accompanied by a long period of quiescence so that the dustecho rings stand out in contrast to the quiescent dust scattering halo. A survey by Valencic \& Smith (2015) showed that a majority of X-ray scattering halos are dominated by scattering from a single cloud, rather than isotropically distributed dust. The time delay associated with a particular angle can be inverted to solve for the angle at which a dust scattering echo will appear $(\theta)$, from a burst that occurred at some time $(t)$ prior to now:

$$
\theta=\left[\frac{2 c(1-x) t}{x D}\right]^{1 / 2}
$$

where $D$ is the distance to the X-ray source, $x$ is the distance to a dust cloud divided by $D$, and $c$ is the speed of light (Trümper \& Schönfelder 1973). This equation can be used to fit dust- 

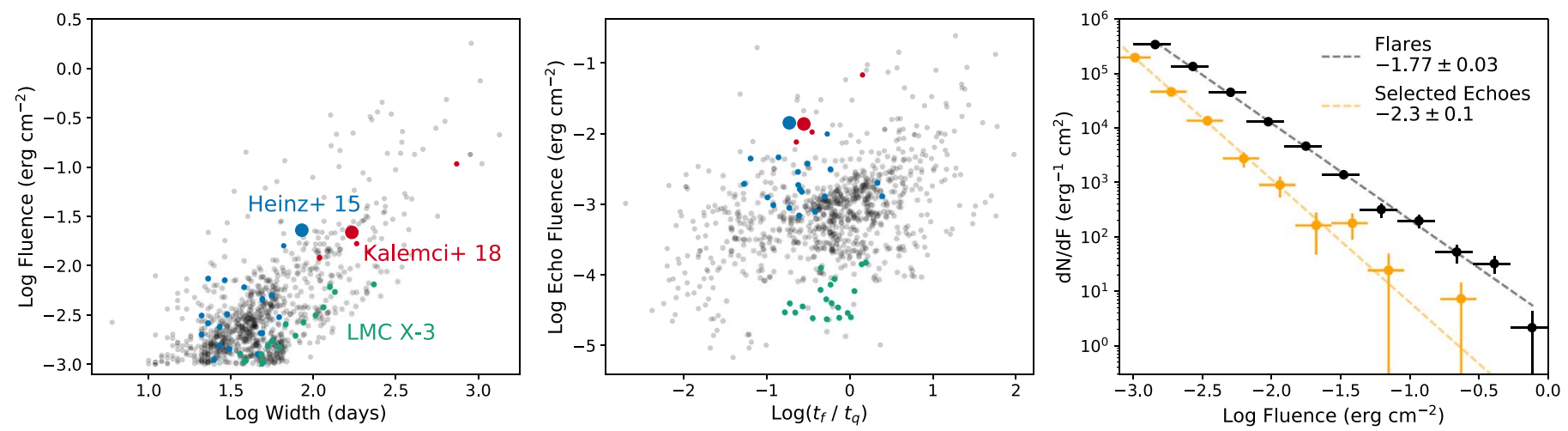

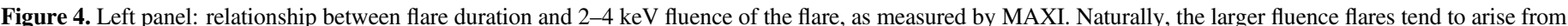

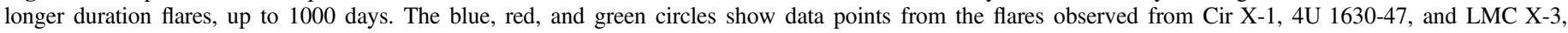

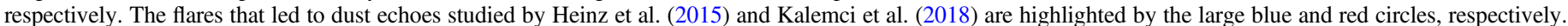

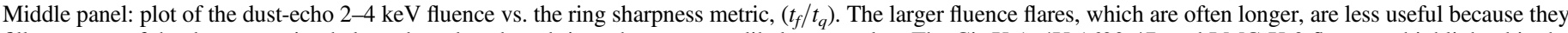

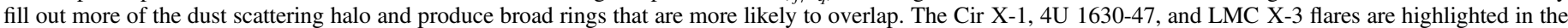

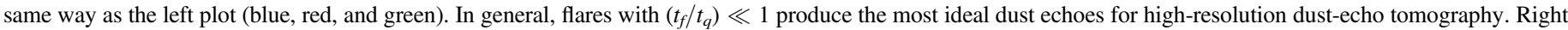

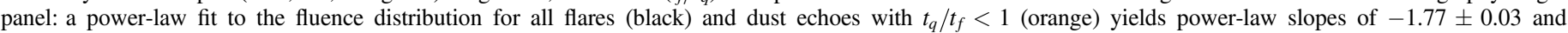
$-2.3 \pm 0.1$, respectively.

echo rings with multiple discrete ISM clouds, or, applying a convolution with the flare light curve, can be used to measure contiguous line-of-sight dust abundances (Heinz et al. 2015, 2016). While clouds or dust material that are extended along the line of sight will alter the perceived thickness and time delay of a dust echo, a full examination of these geometric effects is beyond the scope of this work.

For a fixed dust cloud position, the thickness of a dust-echo ring $(\Delta \theta)$ will depend on the duration of the flare $\left(t_{f}\right)$ so that $\Delta \theta \propto t_{f}^{1 / 2}$. In contrast, the dust scattering halo will return to its quiescent state out to some angle, $\theta \propto t_{q}^{1 / 2}$ where $t_{q}$ is the duration of the quiescent period before or following the flare. We do not set a maximum duration for $t_{f}$. All scattering halos dim in surface brightness at large angular distance from the central source source, so a return to a dim quiescent state will always produce the appearance of rings. This was apparent from the outburst of 4U 1630-47, lasting over 100 days, which produced an 8 arcminute scale ring (Kalemci et al. 2018). Ideal echoes will have thin rings relative to the size of the quiescent halo, requiring $t_{f} / t_{q} \ll 1$.

Figure 4 shows the relationship between flare duration, fluence, and $t_{f} / t_{q}$ for the flares identified in the MAXI data set. We determined the duration of the quiescence directly before and after each flare, and chose the larger $t_{q}$ value. Smaller values of the $t_{f} / t_{q}$ lead to thinner dust-echo rings. The dust echoes arising from the Cir X-1 flare (Heinz et al. 2015) and 4U 1630-47 (Kalemci et al. 2018) were both high fluence with moderate values of $0.1<t_{f} / t_{q}<1$. The flares from LMC X3 are highlighted in Figure 4 (orange) to demonstrate a population of flares arising from a highly variable source with no persistent quiescent state. We found nine objects that produced bright $>10^{-2} \mathrm{erg} \mathrm{cm}^{-2}$ flares detected by MAXI with $\left(t_{f} / t_{q}\right)<0.1$. Of these, four have an estimated dust-echo fluence $>0.005 \mathrm{erg} \mathrm{cm}^{-2}$. These four have not been followed up or published: LS I +61 303, V* BQ Cam, XTE J1752-223, and MAXI J1535-571.

Figure 4 (right) shows the number density of flares as a function of 2-4 keV fluence, which follows a power law of slope $-1.77 \pm 0.03$ (black). We also calculated the fluence distribution for dust echoes with $t_{f} / t_{q}<1$ (orange). The distribution follows a power law for $\log \mathcal{F}_{\text {cgs }}>-3.0$ and is flat for lower fluences, due to our sensitivity limit. For this reason, we limit analysis to the $\log \mathcal{F}_{\text {cgs }}>-3.0$ dust-echo distribution, which fits with a power law of slope $-2.3 \pm 0.1$. In the next section, we use this trend to estimate the number of dust echoes that will be seen by the next generation of X-ray observatories.

\subsection{Avenues for Future Study}

In the future, more sensitive X-ray telescopes will extend dust-echo tomography to dimmer flares, opening up more sight lines for probing the three-dimensional (3D) distribution of dust via X-ray scattering. For a fixed exposure time, we solved for the fluence $(f)$ for which the signal-to-noise ratio is the same as the signal-to-noise ratio for a telescope with no background $\left(f_{0}\right)$,

$$
f=\frac{f_{0}}{2} \times\left(1+\sqrt{1+4 b / f_{0}}\right)
$$

where $b$ is the background surface brightness. ${ }^{13}$ Since any flux threshold is inversely proportional to the effective area $(a)$, we substitute $f_{0}$ with $1 / a$ in Equation (2). We calculated $f$ for a grid of effective areas and backgrounds, relative to Chandra. We then calculated the total number of scattering ring echoes expected $(N)$ by integrating the predicted fluence density distribution for echoes, $d N / d f$ (Figure 4, orange), extrapolating the power law to fluences with $\log \mathcal{F}_{\text {cgs }}<-3$. Figure 5 shows several contours for $N$, predicting the observable number of high signal-to-noise scattering ring echoes, relative to the Chandra effective area and background surface brightness.

Athena, expected to launch around 2030, will have a $1 \mathrm{keV}$ effective area of $2 \mathrm{~m}^{2}$ (Barcons et al. 2017), approximately 80 times the current soft X-ray effective area for Chandra ACIS-I. Athena will thereby observe on the order of 30 times more dust echoes than Chandra can, depending on the instrument

\footnotetext{
13 The background is due to a combination of instrumental, charged particle, and cosmic X-ray background, which change with time and position on the sky. Chandra has relatively low, stable, and well documented background rates compared to other currently active X-ray telescopes, making Chandra a good baseline for comparison.
} 


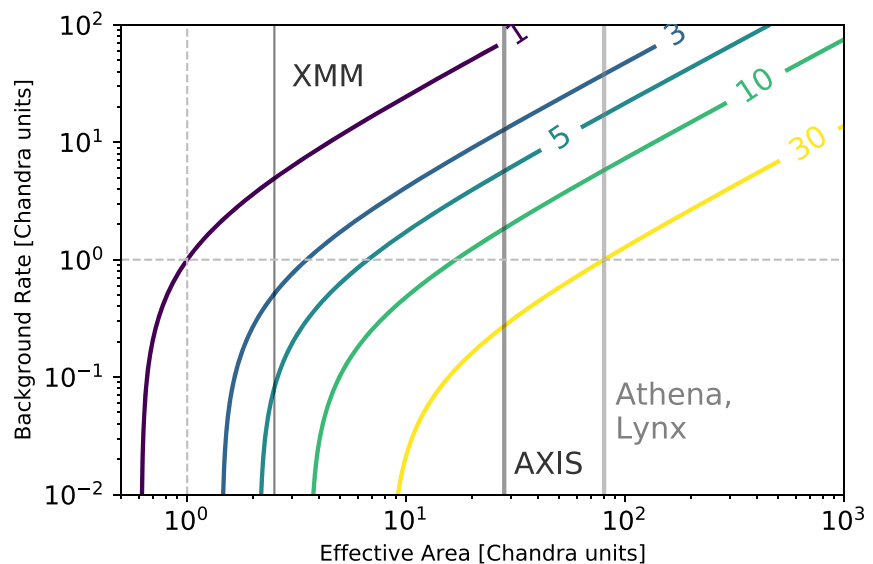

Figure 5. Predicted number of dust scattering echoes observable by different telescopes, depending on the effective area and background levels, as compared to Chandra. For the next generation of telescopes (Athena, Lynx, and AXIS), we expect about 30 times more dust echoes than observable with current instruments, depending on the background levels achieved.

background levels. The concept mission, Lynx, will have a similar effective area to Athena with the imaging resolution of Chandra. The Advanced X-ray Imaging Satellite (AXIS) concept mission has a proposed $1 \mathrm{keV}$ effective area of $7000 \mathrm{~cm}^{2}$ with 10-20 times lower background than Chandra (Mushotzky 2018). Thus, AXIS would be able to image a similar number of dust echoes to Athena and Lynx.

The increased sensitivity offered by the next generation of $\mathrm{X}$-ray telescopes will also constrain the abundance and distribution of dust in the IGM through dust scattering echoes left behind by previously active galactic nuclei (AGN). Using the formulations of Corrales (2015), a telescope with ten times the Chandra sensitivity will be able to image IGM scattering echoes on the order of $20^{\prime \prime}-80^{\prime \prime}$ in radius, corresponding to AGN activity $\sim 10^{2}-10^{3} \mathrm{yr}$ prior. Using the numbers of bright $z>1$ AGN visible from all-sky surveys, the number of echoes one can expect to find in the entire sky is:

$$
N_{\text {ech }}^{\mathrm{IGM}} \sim 10-100\left(\frac{\nu_{f b}}{10^{-3} \mathrm{yr}^{-1}}\right)
$$

where $\nu_{f b}$ is the characteristic frequency for rapid quenching of an AGN accretion flow. We refer the reader to the original work of Corrales (2015) for a detailed discussion on how AGN variability and feedback can be constrained by IGM dust echoes.

\section{Conclusions}

Examination of nine years of MAXI light curves reveals 34 objects that exhibited bright X-ray flares with fluences $>10^{-2} \mathrm{erg} \mathrm{cm}^{-2}$, with durations $\sim 30-300$ days. By comparing the flare duration to the time in quiescence, we estimate that nine of these were short enough to produce sharp ring echoes: approximately one candidate per year. Using $N_{\mathrm{H}}$ to estimate the dust-echo brightness, four of the flares might have produced dust-echo rings detectable by current X-ray telescopes. Only one of these sight lines, Cir X-1, has been imaged and studied in detail.

With the next generation of X-ray telescopes, dust ring echoes will become common features of the Galactic ISM. We expect Athena, Lynx, and AXIS to be $\geqslant 30$ times more sensitive to dust echoes in comparison to Chandra. The result will be hundreds of time-variable X-ray scattering halos. Of these, we expect $\sim 10-30$ sharp dust ring echoes per year, which are ideal for determining 3D distributions of ISM dust with the detail of Heinz et al. (2015, 2016).

Despite this work focusing on thin ring dust echoes, all bright X-ray sources have dust scattering halos that vary with the light curve of the central source. About half of the flares found in this study had $t_{f} / t_{q}>1$. The resulting image will be a blend of broad rings. Interpreting these images will require more advanced dust scattering halo timing techniques. The results will open an avenue for mapping Galactic and intergalactic structures in an entirely new way.

We wish to thank the anonymous referee for their thoughtful comments that greatly improved the clarity of the paper. This research has made use of MAXI data provided by RIKEN, JAXA, and technical support from the MAXI team. Support for this work was provided by NASA through Einstein Postdoctoral Fellowship grant number PF6-170149 awarded by the Chandra X-ray Center (CXC), which is operated by the Smithsonian Astrophysical Observatory for NASA under contract NAS8-03060. Additional support for this work came from CXC through grant No. TM6-17010X.

Facilities: MAXI (Matsuoka et al. 2009).

Software: astropy (Astropy Collaboration et al. 2018), SciPy, NumPy.

\section{ORCID iDs}

Lia Corrales (D) https://orcid.org/0000-0002-5466-3817

Brianna S. Mills (10) https://orcid.org/0000-0003-0148-2817

Sebastian Heinz (ib https://orcid.org/0000-0002-8433-8652

\section{References}

Astropy Collaboration, Price-Whelan, A. M., Sipőcz, B. M., et al. 2018, AJ, 156,123

Barcons, X., Barret, D., Decourchelle, A., et al. 2017, AN, 338, 153

Beardmore, A. P., Willingale, R., Kuulkers, E., et al. 2016, MNRAS, 462, 1847 Corrales, L. 2015, ApJ, 805, 23

Corrales, L., \& Paerels, F. 2012, ApJ, 751, 93

Corrales, L. R., García, J., Wilms, J., \& Baganoff, F. 2016, MNRAS, 458, 1345

Godet, O., Page, K. L., Goad, M. R., et al. 2007, GCNR, 30, 1

Halpern, J. P., Gotthelf, E. V., Reynolds, J., Ransom, S. M., \& Camilo, F. 2008, ApJ, 676, 1178

Heinz, S., Burton, M., Braiding, C., et al. 2015, ApJ, 806, 265

Heinz, S., Corrales, L., Smith, R., et al. 2016, ApJ, 825, 15

Jin, C., Ponti, G., Haberl, F., Smith, R., \& Valencic, L. 2018, MNRAS, 477, 3480

Kalemci, E., Maccarone, T. J., \& Tomsick, J. A. 2018, ApJ, 859, 88

Kawai, N., Tomida, H., Yatsu, Y., et al. 2014, Proc. SPIE, 9144, 91442P

Matsuoka, M., Kawasaki, K., Ueno, S., et al. 2009, PASJ, 61, 999

Miralda-Escudé, J. 1999, ApJ, 512, 21

Mushotzky, R. 2018, Proc. SPIE, 10699, 1069929

Negoro, H., Matsumitsu, T., Mihara, T., et al. 2015, ATel, 7646

Olausen, S. A., Kaspi, V. M., Ng, C.-Y., et al. 2011, ApJ, 742, 4

Pintore, F., Mereghetti, S., Tiengo, A., et al. 2017a, MNRAS, 467, 3467

Pintore, F., Tiengo, A., Mereghetti, S., et al. 2017b, MNRAS, 472, 1465

Predehl, P., \& Schmitt, J. H. M. M. 1995, A\&A, 293, 889

Tiengo, A., \& Mereghetti, S. 2006, A\&A, 449, 203

Tiengo, A., Vianello, G., Esposito, P., et al. 2010, ApJ, 710, 227

Trümper, J., \& Schönfelder, V. 1973, A\&A, 25, 445

Valencic, L. A., \& Smith, R. K. 2015, ApJ, 809, 66

Vasilopoulos, G., \& Petropoulou, M. 2016, MNRAS, 455, 4426

Vaughan, S., Willingale, R., O’Brien, P. T., et al. 2004, ApJL, 603, L5

Vaughan, S., Willingale, R., Romano, P., et al. 2006, ApJ, 639, 323

Vianello, G., Tiengo, A., \& Mereghetti, S. 2007, A\&A, 473, 423

Watson, D., Vaughan, S. A., Willingale, R., et al. 2006, ApJ, 636, 967 